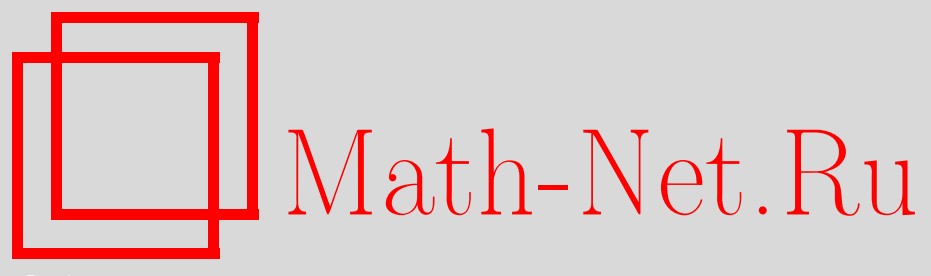

И. А. Шейпак, Особые точки самоподобной функции нулевого спектрального порядка. Самоподобная струна Стилтьеса, Матем. заметки, 2010, том 88, выпуск $2,303-316$

DOI: https://doi.org/10.4213/mzm5264

Использование Общероссийского математического портала Math-Net.Ru подразумевает, что вы прочитали и согласны с пользовательским соглашением http://www . mathnet.ru/rus/agreement

Параметры загрузки:

IP: 54.197 .217 .227

26 апреля 2023 г., 18:13:37

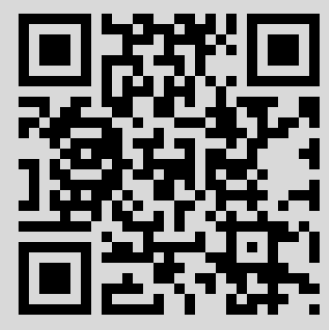


Том 88 выпуск 2 август 2010

УДК $517.518+517.984$

\section{Особые точки самоподобной функции нулевого спектрального порядка. Самоподобная струна Стилтьеса}

\section{И. А. Шейпак}

В статье вводится понятие самоподобной функции нулевого спектрального порядка и изучаются ее свойства. Эта функция имеет не более чем счетное число точек разрыва, причем все точки разрыва являются точками разрыва первого рода, за исключением, быть может, одной точки, являющейся особой. Получена формула, позволяющая по параметрам самоподобия функции, вычислить ее координаты. Исследуется поведение самоподобной функции в окрестности особой точки.

Неубывающая функция $f$ нулевого спектрального порядка, принадлежащая пространству $L_{2}[0,1]$, порождает самоподобную струну Стилтьеса, т.е. спектральную задачу вида

$$
-y^{\prime \prime}-\lambda \rho y=0, \quad y(0)=y(1)=0,
$$

где $\rho$ есть функция из пространства $\stackrel{\circ}{W_{2}^{-1}}[0,1]$ и $f^{\prime}=\rho$. Незнакоопределенная функция $f$ приводит к понятию самоподобной индефинитной струны Стилтьеca.

Библиография: 13 названий.

\section{1. Введение}

Струна называется стилтъесовской, если она представляет собой невесомую нить, которая несет лишь сосредоточенные массы $m_{1}, m_{2}, \ldots$ в точках $0=x_{1}<x_{2}<\cdots$, сгущающихся к правому концу, $\lim x_{n}=L(\leqslant \infty)$. Задача о колебаниях стилтьесовской струны единичной длины с закрепленными концами приводит к спектральной задаче

$$
\begin{aligned}
& -y^{\prime \prime}-\lambda \rho y=0, \\
& y(0)=y(1)=0,
\end{aligned}
$$

где $\rho=\sum_{k=1}^{\infty} m_{k} \delta\left(x-x_{k}\right)$. Обобщенная первообразная $f\left(f^{\prime}=\rho\right)$ такого веса представляет собой кусочно-постоянную функцию, точки разрыва которой стремятся к правому концу отрезка, а $f(1-0)=\sum_{k=1}^{n} m_{k}(\leqslant \infty)$. С этой точки зрения, можно считать, что точка $x=1$ является особой точкой функции $f$.

Работа выполнена при поддержке Российского фонда фундаментальных исследований (гранты №№ 10-01-00423, 09-01-90408).

(С) И. А. ШЕйпАК, 2010 
Более подробно о стилтьесовской струне см. в [1].

Укажем на более широкий класс спектральных задач, связанных со стилтьесовской струной. В работе [2] изучалась задача (1.1), (1.2) в предположении, что вес $\rho$ является самоподобной мерой. Но случай дискретных мер, т.е. как раз случай стилтьесовской струны не рассматривается. Для таких классов весов были получены асимптотические формулы распределения собственных значений, имеющие степенной порядок.

Самоподобные функции положительного спектрального порядка были введены в работах [3], [4]. Оказывается, что спектральный порядок функции $f$ непосредственно связан с порядком асимптотики собственных значений задачи (1.1), (1.2), где $\rho=f^{\prime}$. В этих работах изучалась задача (1.1),(1.2) с более общим классом весовых функций. А именно, в качестве веса $\rho$ рассматривались самоподобные обобщенные функции из пространства $\stackrel{\circ}{W}-1[0,1]$. Первообразные таких весов являются самоподобными функциями из пространства $L_{2}[0,1]$. При этом условия, накладываемые на параметры самоподобия, исключают из рассмотрения кусочно-постоянные функции, т.е. и в этих работах стилтьесовские струны не рассматривались. Заметим, что собственные значения задачи (1.1), (1.2) с весом, являющимся обобщенной производной функции положительного спектрального порядка, также подчиняются степенному закону распределения.

С точки зрения задачи (1.1), (1.2) случай, когда вес является обобщенной производной самоподобной функции нулевого спектрального порядка, занимает особое место. А именно, собственные значения имеют экспоненциальное распределение (см. [5]). Поэтому изучение таких функций представляет интерес с точки зрения спектральной теории операторов.

Отметим, что самоподобные функции (в том числе и кусочно-постоянные) имеют важное значение не только для спектральных задач. Например, одним из популярных приложений самоподобных непрерывных функций является теория сжатия компьютерных изображений (см. [6]). Различные задачи, связанные с изучением таких свойств самоподобных функций как ограниченность вариации, абсолютная непрерывность и других свойств, естественным образом приводящих к понятию вероятностных самоподобных мер, возникают в теории фрактальных кривых, изучение которых началось с уже ставших классическими работ [7]-[9]. Среди более поздних работ, посвященных различным свойствам фрактальных кривых, отметим работы [10] и [11]. Конструкция аффинно-самоподобных функций, принадлежащих пространствам $L_{p}[0,1]$, приведена в [12]. Кроме того, самоподобные меры находят применение и в теории случайных процессов (см., например, [13]).

Целью статьи является определение функции нулевого спектрального порядка, исследование ее структуры, классификация ее особых точек в зависимости от параметров самоподобия и исследование поведения самоподобной функции в окрестности этой точки.

Также вводится понятие самоподобной струны Стилтьеса, имеющей тесную связь с функциями нулевого спектрального порядка. В отличие от классической струны функции нулевого спектрального порядка порождают, вообще говоря, индефинитную струну (ср. [1], замечание 2.2 , теорема 2.4).

Напомним основные понятия, связанные с самоподобными функциями. 
2. Самоподобные функции в пространстве $L_{p}[0,1]$ и их спектральный порядок

2.1. Операторы подобия в пространстве $L_{p}[0,1]$. Пусть фиксировано натуральное число $n>1$, и пусть вещественные числа $a_{k}>0$, где $k=1, \ldots, n$, таковы, что

$$
\sum_{k=1}^{n} a_{k}=1
$$

а вещественные числа $d_{k}$ и $\beta_{k}$ пока произвольны. Дополнительно определим числа

$$
\alpha_{1}=0, \quad \alpha_{k}=\sum_{l=1}^{k-1} a_{l}, \quad k=2, \ldots, n+1 .
$$

Таким образом, числа $0=\alpha_{1}<\alpha_{2}<\cdots<\alpha_{n}<1=\alpha_{n+1}$ образуют разбиение отрезка $[0,1]$.

Определим непрерывные операторы $G_{k}$ и $\widetilde{G}_{k}$, сопоставляющие функции $f \in$ $L_{p}[0,1]$ функцию из $L_{p}\left[\alpha_{k}, \alpha_{k+1}\right], k=1,2, \ldots, n$, по правилу

$$
\begin{array}{llll}
G_{k}(f)(x)=f(t), & \text { где } \quad x=a_{k} t+\alpha_{k}, & x \in\left[\alpha_{k}, \alpha_{k+1}\right], & t \in[0,1], \\
\widetilde{G}_{k}(f)(x)=f(t), & \text { где } \quad x=-a_{k} t+\alpha_{k+1}, & x \in\left[\alpha_{k}, \alpha_{k+1}\right], & t \in[0,1] .
\end{array}
$$

Окончательно построим нелинейный непрерывный оператор $G: L_{p}[0,1] \rightarrow L_{p}[0,1]$ вида

$$
G(f)=\sum_{k=1}^{n}\left\{\beta_{k} \cdot \chi_{\left(\alpha_{k}, \alpha_{k+1}\right)}+d_{k} \cdot \widehat{G}_{k}(f)\right\},
$$

где $\chi_{\left(\alpha_{k}, \alpha_{k+1}\right)}$ - характеристическая функция интервала $\left(\alpha_{k}, \alpha_{k+1}\right)$, а $\widehat{G}_{k}=G_{k}$ или $\widehat{G}_{k}=\widetilde{G}_{k}$ в зависимости от $k$. Про отображения $\widetilde{G}_{k}$ будем говорить, что они меняют ориентацию отрезка $\left[\alpha_{k}, \alpha_{k+1}\right]$.

Операторы $G$ вида (2.1) будут называться операторами подобия.

В работе [12] рассмотрены более общие операторы подобия

$$
G(f)=\sum_{k=1}^{n}\left\{\beta_{k} \cdot \chi_{\left(\alpha_{k}, \alpha_{k+1}\right)}+c_{k} \cdot x+d_{k} \cdot G_{k}(f)\right\},
$$

но не рассматривались операторы, меняющие ориентацию отрезков $\left[\alpha_{k}, \alpha_{k+1}\right]$.

Там же доказана следующая

Лемма 2.1. Оператор подобия $G$ является сжимающим в $L_{p}[0,1]$ в том и только том случае, когда справедливы неравенства

$$
\begin{array}{cc}
\sum_{k=1}^{n} a_{k}\left|d_{k}\right|^{p}<1, & 1 \leqslant p<+\infty, \\
\max _{1 \leqslant k \leqslant n}\left|d_{k}\right|<1, \quad & p=+\infty .
\end{array}
$$

Из леммы 2.1 и принципа сжимающих отображений немедленно вытекает справедливость следующего утверждения. 
Теорема 2.1. Если справедливо неравенство (2.3) (либо (2.4)), то существует u единственна функция $f \in L_{p}[0,1]$, удовлетворяющая уравнению $G(f)=f$.

В дальнейшем всегда будет предполагаться, что либо неравенство (2.3), либо неравенство (2.4) выполнено.

Если функция $f \in L_{p}[0,1]$ удовлетворяет уравнению $G(f)=f$, где $G$ - некоторый оператор подобия, то такая функция будет называться самоподобной. При этом величины $n, a_{k}, d_{k}$ и $\beta_{k}$, где $k=1,2, \ldots, n$, определяющие соответствующий оператор подобия $G$, будут называться параметрами самоподобия функции $f$.

2.2. Спектральный порядок. Заметим, что если для всех $k=1,2, \ldots, n$ выполнено $\beta_{k}=0$, то оператор подобия $G$ имеет только тривиальную неподвижную точку $f \equiv 0$, поэтому в дальнейшем будем предполагать, что выполнено

УСловие $(B)$. Среди чисел $\beta_{k}$, где $k=1, \ldots, n$, по меньшей мере одно отлично от нуля.

Среди самоподобных функций, удовлетворяющих условию $(B)$, выделим следующие классы, для которых параметры самоподобия соответственно обладают следующими свойствами.

УСловиЕ $\left(D_{0}\right)$. Выполнено $d_{k}=0$ для всех $k=1, \ldots, n$.

УСловиЕ $\left(D_{1}\right)$. Среди чисел $d_{k}$, где $k=1, \ldots, n$, ровно одно отлично от нуля.

Условие $\left(D_{2}\right)$. Среди чисел $d_{k}$, где $k=1, \ldots, n$, не менее двух отличны от нуля.

Самоподобные функции, принадлежащие пространству $L_{2}[0,1]$ и удовлетворяющие условию $\left(D_{2}\right)$, были введены в работах [3], [4] и называются самоподобными функииями положительного спектрального порядка. В этой же работе показано, что для параметров самоподобия, отвечающих такой функции, уравнение

$$
\sum_{k=1}^{n}\left(a_{k}\left|d_{k}\right|\right)^{D}=1
$$

имеет решение $0<D<1$, а для собственных значений $\lambda_{n}$ задачи (1.1), (1.2) справедлива асимптотическая формула

$$
\left|\lambda_{n}\right| \asymp n^{1 / D} .
$$

Нетрудно заметить, что самоподобные функции, удовлетворяющие условию $\left(D_{0}\right)$, суть простые функции, принимающие конечное число значений, а именно,

$$
f=\sum_{k=1}^{n} \beta_{k} \cdot \chi_{\left(\alpha_{k}, \alpha_{k+1}\right)} .
$$

Обобщенная производная такой функции имеет вид

$$
f^{\prime}(x)=\sum_{k=2}^{n}\left(\beta_{k}-\beta_{k-1}\right) \cdot \delta\left(x-\alpha_{k}\right) .
$$

Подстановка (2.6) в качестве веса $\rho$ в (1.1), (1.2) сводит задачу к конечномерной. Непосредственное вычисление позволяет убедиться, что собственные значения 
в этом случае являются корнями многочлена

$$
\begin{aligned}
& 1-\lambda \sum_{k=2}^{n} m_{k} \alpha_{k}\left(1-\alpha_{k}\right)+\lambda^{2} \sum_{2 \leqslant i_{1}<i_{2} \leqslant n} m_{i_{1}} m_{i_{2}} \alpha_{i_{1}}\left(\alpha_{i_{2}}-\alpha_{i_{1}}\right)\left(1-\alpha_{i_{2}}\right)+\cdots \\
& +(-1)^{k} \lambda^{k} \sum_{2 \leqslant i_{1}<i_{2}<\cdots<i_{k} \leqslant n}^{m_{i_{1}} m_{i_{2}} \cdots m_{i_{k}}} \\
& \quad \times \alpha_{i_{1}}\left(\alpha_{i_{2}}-\alpha_{i_{1}}\right) \cdots\left(\alpha_{i_{k}}-\alpha_{i_{k-1}}\right)\left(1-\alpha_{i_{2}}\right)+\cdots \\
& \quad+(-1)^{n-1} \lambda^{n-1} m_{2} m_{3} \cdots m_{n} \alpha_{2}\left(\alpha_{3}-\alpha_{2}\right) \cdots\left(\alpha_{n}-\alpha_{n-1}\right)\left(1-\alpha_{n}\right),
\end{aligned}
$$

где $m_{k}=\beta_{k}-\beta_{k-1}, k=2,3, \ldots, n$.

Для самоподобных функций класса $\left(D_{1}\right)$ уравнение $(2.5)$ имеет только тривиальное решение $D=0$. В связи с этим дадим следующее

ОПРеДЕЛЕНИЕ 2.1. Самоподобные функции класса $D_{1}$ будем называть самоподобными функциями нулевого спектрального порядка.

Изучим более подробно функции класса $D_{1}$ (не обязательно принадлежащие пространству $\left.L_{2}[0,1]\right)$. Обозначим через $\widehat{k}$ тот единственный индекс, для которого $d_{\widehat{k}} \neq 0,1 \leqslant \widehat{k} \leqslant n$.

Справедливо следующее утверждение.

ТЕОРема 2.2. Самоподобная функиия нулевого спектрального порядка является кусочно-постоянной и принимает не более чем счетное число значений. Все точки разрыва являются точками разрыва первого рода, кроме, быть может, одной точки.

ДокАЗАТЕЛЬство. Рассмотрим сначала случай, когда в формуле (2.1) все операторы $\widehat{G}_{k}$ равны $G_{k}$. Случай, когда при некоторых $k \in[1, n]$ оператор $\widehat{G}_{k}=\widetilde{G}_{k}$, рассматривается аналогично. Функция $f$, являющаяся неподвижной точкой оператора $G$, в этом случае имеет некоторые отличия, о которых мы расскажем в разделе 3 .

Для каждого натурального $m$ построим разбиение отрезка $[0,1]$ на $n^{m}$ отрезков и каждому такому отрезку разбиения сопоставим последовательность чисел $k_{1}, k_{2}, \ldots, k_{m}$ (номер отрезка), где $k_{i}=1,2, \ldots, n, i=1,2, \ldots, m$.

При $m=1$ такое разбиение образуют отрезки

$$
I_{k_{1}}:=\left[\alpha_{k_{1}}, \alpha_{k_{1}+1}\right], \quad k_{1}=1,2, \ldots, n .
$$

Такие отрезки будем называть отрезками разбиения первого порядка или просто отрезками первого порядка.

При $m=2$ разбиение образуют отрезки

$$
I_{k_{1}, k_{2}}:=\left\{y=a_{k_{2}} x+\alpha_{k_{2}}, x \in I_{k_{1}}\right\}, \quad k_{i}=1,2, \ldots, n,
$$

где $i=1,2$. Такие отрезки будем называть отрезками разбиения второго порядка или просто отрезками второго порядка.

Далее построение осуществляется индуктивно. Если построено разбиение на отрезки $I_{k_{1}, k_{2}, \ldots, k_{m-1}}, k_{i}=1,2, \ldots, n, i=1,2, \ldots, m-1$, то очередное разбиение определяется следующим образом:

$$
I_{k_{1}, k_{2}, \ldots, k_{m}}:=\left\{y=a_{k_{m}} x+\alpha_{k_{m}}, x \in I_{k_{1}, k_{2}, \ldots, k_{m-1}}\right\}, \quad k_{i}=1,2, \ldots, n,
$$


где $i=1,2, \ldots, m$. Такие отрезки будем называть отрезками разбиения $m$-го порядка или просто отрезками $m$-го порядка. Нетрудно заметить, что отрезок $I_{k_{1}, k_{2}, \ldots, k_{m}}$ $m$-го порядка имеет длину $a_{k_{1}} a_{k_{2}} \cdots a_{k_{m}}$.

Пусть $f_{0} \equiv 0$. Построим последовательность $f_{j}, j=1,2, \ldots$, по правилу $f_{j}=$ $G\left(f_{j-1}\right)$. Очевидно, что $f_{j} \rightarrow f$ в $L_{p}[0,1]$. При этом в силу соотношения $(2.1)$ выполнено

$$
\begin{aligned}
& f_{1}(x)= \beta_{k_{1}} x \in I_{k_{1}}, \quad k_{1}=1,2, \ldots, n, \\
& f_{2}(x)= \begin{array}{ll}
\beta_{k_{1}}, & x \in I_{k_{1}}, \quad k_{1} \neq \widehat{k}, \\
d_{\widehat{k}} \beta_{k_{2}}+\beta_{\widehat{k}}, \quad x \in I_{\widehat{k}, k_{2}}, \quad k_{2}=1,2, \ldots, n,
\end{array} \\
& f_{3}(x)= \begin{cases}\beta_{k_{1}}, & x \in I_{k_{1}}, \quad k_{1} \neq \widehat{k}, \\
d_{\widehat{k}} \beta_{k_{2}}+\beta_{\widehat{k}}, & x \in I_{\widehat{k}, k_{2}}, \quad k_{2} \neq \widehat{k}, \\
d_{\widehat{k}}\left(d_{\widehat{k}} \beta_{k_{3}}+\beta_{\widehat{k}}\right)+\beta_{\widehat{k}}, \quad x \in I_{\widehat{k}, \widehat{k}, k_{3}}, \quad k_{3}=1,2, \ldots, n,\end{cases} \\
& \ldots
\end{aligned}
$$

Несложно заметить, что функции $f_{m}$ обладают тем свойством, что вне отрезка $I_{\widehat{\widehat{k}}, \widehat{k}, \ldots, \widehat{k}}$ имеет место равенство

$$
G\left(f_{m}\right)=f_{m}
$$

где равенство выполнено в смысле пространства $L_{p}[0,1]$. Следовательно, вследствие

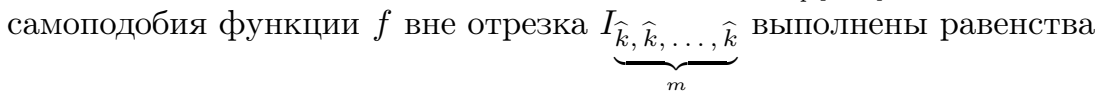

$$
f_{m}=f \text {. }
$$

Таким образом, функции $f_{m}$ равны функции $f$ вне одного отрезка $m$-го порядка.

Отрезки $\underbrace{I_{m}}_{\widehat{c}, \widehat{k}, \ldots, \widehat{k}}$ образуют систему вложенных отрезков, стягивающихся к точке $\widehat{x}$, являющейся в некотором роде особой для самоподобной функции $f$. Левый $\left(x_{l}\right)$ и правый $\left(x_{r}\right)$ концы отрезка $\underbrace{I_{m}}_{\widehat{c}, \widehat{k}, \ldots, \widehat{k}}$ соответственно имеют координаты

$$
\begin{aligned}
x_{l} & =\alpha_{\widehat{k}}+a_{\widehat{k}} \alpha_{\widehat{k}}+a_{\widehat{k}}^{2} \alpha_{\widehat{k}}+\cdots+a_{\widehat{k}}^{m-1} \alpha_{\widehat{k}}, \\
x_{r} & =\alpha_{\widehat{k}}+a_{\widehat{k}} \alpha_{\widehat{k}}+a_{\widehat{k}}^{2} \alpha_{\widehat{k}}+\cdots+a_{\widehat{k}}^{m-2} \alpha_{\widehat{k}}+a_{\widehat{k}}^{m-1} \alpha_{\widehat{k}+1} .
\end{aligned}
$$


Координату точки $\widehat{x}$ можно вычислить по формуле

$$
\widehat{x}=\frac{\alpha_{\widehat{k}}}{1-a_{\widehat{k}}} .
$$

В частности, если $\widehat{k}=n$, т.е. ровно последнее $d_{k}$ отлично от нуля, то из $(2.11)$ и определений чисел $\left\{a_{k}\right\},\left\{\alpha_{k}\right\}$ вытекает, что $\widehat{x}=1$. Соответственно, при $\widehat{k}=1$ получаем, что $\widehat{x}=0$.

Если $x \neq \widehat{x}$, то из соотношений (2.7)-(2.10) следует, что существуют правые и левые пределы функции $f$ - соответственно $f(x+0)$ и $f(x-0)$. Причем $f(x+0) \neq$ $f(x-0)$ не более чем в счетном числе точек. Этими точками могут быть только концы отрезков $m$-го порядка при $m=1,2, \ldots$. Но не все концы указанных отрезков обязательно являются точками разрыва функции $f$.

Укажем тривиальный случай, при котором функция, формально принадлежащая классу $\left(D_{1}\right)$, принимает конечное число значений. А именно, это возможно, только если

$$
\beta_{1}=\beta_{2}=\cdots=\beta_{\widehat{k}-1}=\beta_{\widehat{k}+1}=\cdots=\beta_{n}=d_{\widehat{k}} \beta_{1}+\beta_{\widehat{k}},
$$

что следует из соотношения для $f_{2}$ в формуле (2.8). Самоподобная функция в этой ситуации тождественно равна константе $\beta_{1}$.

В частности, при $n=2$ (без ограничения общности считаем, что $\widehat{k}=2$ ), эти условия сводятся к равенству $d_{2} \beta_{1}+\beta_{2}=\beta_{1}$. Теорема 2.2 доказана.

ЗАмечАниЕ 2.1. Всегда будем предполагать, что при $i, i+1 \neq \widehat{k}$ выполнено $\beta_{i} \neq \beta_{i+1}$. Иначе, можно уменьшить параметр $n$ на единицу, объединив преобразования $\widehat{G}_{i}$ и $\widehat{G}_{i+1}$ в одно новое преобразование $\widehat{G}_{i}$. Из формул $(2.7)-(2.10)$ следует, что при $i \neq \widehat{k}$ операторы $G_{i}$ и $\widetilde{G}_{i}$ действуют одинаково.

В окрестности точки $\widehat{x}$ поведение самоподобной функции $f$ может быть более сложным. Опираясь на формулы (2.10), разберем эти случаи подробнее.

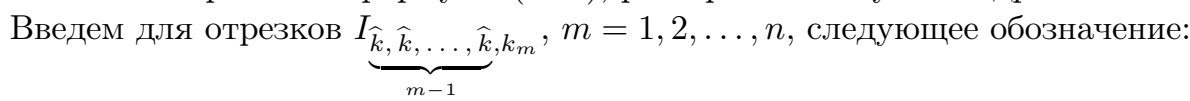

$$
I\left(m, k_{m}\right):=I_{\widehat{k}, \widehat{k}, \ldots, \widehat{k}}^{I_{m-1}}, k_{m} .
$$

Из соотношений $(2.10)$ следует, что для $i \neq \widehat{k}$ справедливы соотношения

$$
\begin{array}{ll}
\left.f\right|_{I(m, i)}=d_{\widehat{k}}^{m-1}\left(\frac{\beta_{\widehat{k}}}{d_{\widehat{k}}-1}+\beta_{i}\right)-\frac{\beta_{\widehat{k}}}{d_{\widehat{k}}-1} & \text { при } \quad d_{\widehat{k}} \neq 1 \\
\left.f\right|_{I(m, i)}=(m-1) \beta_{\widehat{k}}+\beta_{i} & \text { при } \quad d_{\widehat{k}}=1 .
\end{array}
$$

Из этих формул следует, что в окрестности точки $\widehat{x}$ возможно следующее поведение функции $f$.

1. Случай $\left|d_{\widehat{k}}\right|<1$. В этом случае функция $f$ принадлежит пространству $B[0,1]$ (ограниченных на отрезке функций). Доказательство этого факта полностью аналогично доказательству теоремы 2.1 работы [12]. При этом, существует предел значений функции $f$ в точке $\widehat{x}$ и

$$
\lim _{x \rightarrow \widehat{x}} f(x)=\frac{\beta_{\widehat{k}}}{1-d_{\widehat{k}}} .
$$


Пример такой функции представлен на рис. 1 , где $n=2, a_{1}=a_{2}=0.5, d_{1}=$ $\beta_{1}=0, d_{2}=\beta_{2}=0.5, \widehat{x}=1$.

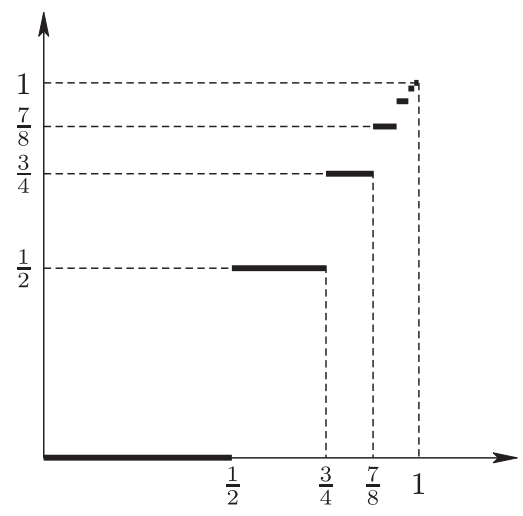

Рис. 1. Пример 1

2. Случай $d_{\widehat{k}}=1$. Здесь возможны три случая (исключая тривиальный, указанный в доказательстве теоремы 2.2).

а) Если $\beta_{\widehat{k}}=0, n=3, \widehat{k}=2$, то функция $f$ принимает конечное число значений и $\widehat{x}$ является точкой разрыва первого рода. При этом $f(\widehat{x}-0)=\beta_{1}, f(\widehat{x}+0)=\beta_{3}$.

б) Если $\beta_{\widehat{k}}=0$ и не имеет место ситуация, описанная в $\left.2, \mathrm{a}\right)$, то $\widehat{x}$ является точкой разрыва второго рода.

Пример такой функции представлен на рис. 2 , где $n=3, a_{1}=0.5, a_{2}=a_{3}=0.25$, $d_{3}=\beta_{1}=1, \beta_{2}=\beta_{3}=0, \widehat{x}=1$.



Рис. 2. Пример 2, б)

в) Если $\beta_{\widehat{k}} \neq 0$, то

$$
\lim _{x \rightarrow \widehat{x}} f(x)=\operatorname{sign} \beta_{\widehat{k}} \cdot(+\infty) .
$$

3. Случай $d_{\widehat{k}}>1$. Здесь возможны два случая. 
а) Если все выражения $\beta_{\widehat{k}} /\left(d_{\widehat{k}}-1\right)+\beta_{j}, j \neq \widehat{k}$, имеют один и тот же знак, то существует

$$
\lim _{x \rightarrow \widehat{x}} f(x)=\operatorname{sign}\left(\frac{\beta_{\widehat{k}}}{d_{\widehat{k}}-1}+\beta_{j}\right) \cdot(+\infty) .
$$

Пример такой функции представлен на рис. 3 , где $n=3, a_{1}=a_{2}=a_{3}=1 / 3$, $\beta_{1}=\beta_{2}=1, \beta_{3}=0, d_{2}=2, \widehat{x}=0.5$.

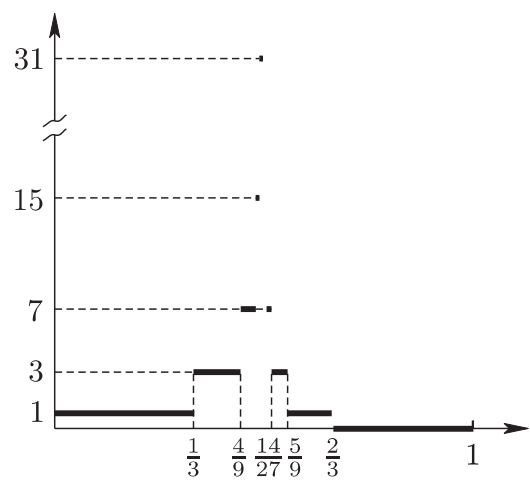

Рис. 3. Пример 3, а)

б) Если выражения $\beta_{\widehat{k}} /\left(d_{\widehat{k}}-1\right)+\beta_{j}$ при $j \neq \widehat{k}$ имеют разные знаки, то $\widehat{x}-$ точка разрыва второго рода функции $f$. Нулевое значение в этом случае считается отличным и от положительного, и от отрицательного значений.

Заметим, что если $\widehat{k}=2$ и $d_{2} \beta_{1}+\beta_{2}=\beta_{1}$, то существует $f(\widehat{x}-0)=\beta_{1}$. Аналогично, если $\widehat{k}=n-1$ и $d_{n-1} \beta_{n}+\beta_{n-1}=\beta_{n}$, то существует $f(\widehat{x}+0)=\beta_{n}$.

Пример такой функции представлен на рис. 4 , где $n=3, a_{1}=a_{2}=a_{3}=1 / 3$, $\beta_{1}=\beta_{2}=1, \beta_{3}=-2, d_{2}=2, \widehat{x}=0.5$.

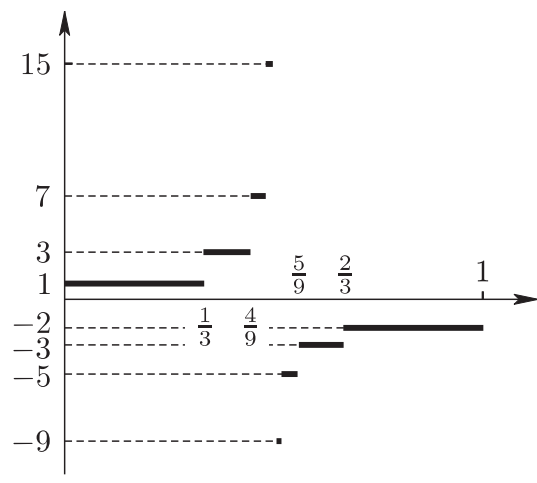

Рис. 4. Пример 3, б)

4. Случай $d_{\widehat{k}} \leqslant-1$. В этом случае $\widehat{x}$ - точка разрыва второго рода.

ОПРЕДЕЛЕНИЕ 2.2. Самоподобной струной Стилтъеса будем называть струну, колебания которой описываются задачей $(1.1),(1.2)$, где вес $\rho$ представляет собой обобщенную производную самоподобной функции класса $\left(D_{1}\right)$. 
ЗАМЕчАНИЕ 2.2. Из соотношения (2.11) следует, что в отличие от классической стилтьесовской струны точка накопления масс не обязательно является концом нити. Кроме того, в этом случае задача может быть индефинитной, т.е. "массы" могут принимать отрицательное значение.

В связи с этим представляет интерес вопрос о нахождении условий на параметры самоподобия, при которых они порождают неубывающую самоподобную функцию. Для произвольной самоподобной функции, являющейся неподвижной точкой оператора $G$, определенного формулой (2.2), в работе [12] получены достаточные условия неубывания функции $f$. Заметим, что в этой работе в формулировке теоремы о достаточных условиях неубывания самоподобной функции допущена ошибка. Для восстановления правильного утверждения и для полноты изложения приведем формулировку этого результата (см. теорему 4.1 [12]).

ТЕОрема 2.3. Пусть непрерывная слева функиия $f$ является неподвижной точкой сжимающего оператора $G$, определенного формулой (2.2), а параметры самоподобия $\left\{c_{k}\right\},\left\{d_{k}\right\}$ u $\left\{\beta_{k}\right\}$ выбраны так, что $f(0)=1$ u $f(1)=1$. Тогда для того, чтобы функиия $f$ была неубывающей, необходимо, чтобы при всех $k=1,2, \ldots, n$ выполнялись неравенства

$$
\beta_{k} \leqslant \beta_{k+1}, \quad c_{k}+d_{k}+\beta_{k} \leqslant \beta_{k+1}, \quad c_{k}+d_{k} \geqslant 0,
$$

и достаточно, чтобъ при всех $k=1,2, \ldots, n$ выполнялись неравенства

$$
\beta_{k} \leqslant \beta_{k+1}, \quad c_{k}+d_{k}+\beta_{k} \leqslant \beta_{k+1}, \quad c_{k} \geqslant 0, \quad d_{k} \geqslant 0 .
$$

В общей ситуации между необходимыми и достаточными условиями есть “зазор”. В работе [12] приведен пример самоподобной функции, удовлетворяющей условиям (2.12), но не являющейся монотонной.

Для самоподобной функции нулевого спектрального порядка можно сформулировать критерий монотонности в терминах параметров самоподобия.

ТЕОрема 2.4. Самоподобная функиия $f \in D_{1}$ является неубывающей тогда и только тогда, когда ее параметры самоподобия удовлетворяют следующим условиям:

1) $d_{\widehat{k}}>0$

2) выполнено

$$
\beta_{1} \leqslant \cdots \leqslant \beta_{\widehat{k}-1} \leqslant d_{\widehat{k}} \beta_{1}+\beta_{\widehat{k}} \leqslant d_{\widehat{k}} \beta_{n}+\beta_{\widehat{k}} \leqslant \beta_{\widehat{k}+1} \leqslant \cdots \leqslant \beta_{n}, \quad 1<\widehat{k}<n .
$$

При $\widehat{k}=n$ условие 2) меняется на неравенства

$$
\beta_{1} \leqslant \cdots \leqslant \beta_{n-1} \leqslant \beta_{n-1} \leqslant d_{n} \beta_{1}+\beta_{n} .
$$

При $\widehat{k}=1$ условие 2) меняется на неравенства

$$
d_{1} \beta_{n}+\beta_{1} \leqslant \beta_{2} \leqslant \cdots \leqslant \beta_{n}
$$

Чтобы функиия $f$ не возрастала, неравенства 2) надо поменять на противоположные. 
ДокАЗАтЕЛЬСтво. Рассмотрим случай $1<\widehat{k}<n$. Из формулы $(2.7)$ следует, что для неубывания самоподобной функции на отрезках первого порядка необходимо и достаточно, чтобы выполнялись неравенства $\beta_{i} \leqslant \beta_{j}$ для всех $i<j, i, j \neq \widehat{k}$. Чтобы функция не убывала на отрезках второго порядка, необходимо и достаточно, чтобы она не убывала при переходе с отрезка первого порядка с номером $\widehat{k}-1$ на первый отрезок второго порядка с номером $\widehat{k}, 1$ и при переходе с последнего отрезка второго порядка с номером $\widehat{k}, n$ на отрезок первого порядка с номером $\widehat{k}+1$. Из формул $(2.8)$ следует, что для этого необходимо и достаточно выполнения неравенств

$$
\beta_{\widehat{k}-1} \leqslant d_{\widehat{k}} \beta_{1}+\beta_{\widehat{k}}, \quad d_{\widehat{k}} \beta_{n}+\beta_{\widehat{k}} \leqslant \beta_{\widehat{k}+1} .
$$

В силу самоподобия функции $f$ условия неубывания будут выполняться и на отрезках более высокого порядка, что непосредственно следует из неравенств (2.8)$(2.10)$.

Необходимость условия $d_{\widehat{k}}>0$ очевидна.

Случаи $\widehat{k}=1$ и $\widehat{k}=n$ рассматриваются аналогично. Теорема 2.4 доказана.

Легко видеть, что для самоподобной функции $f$ нулевого спектрального порядка, удовлетворяющей условиям $f(0)=0, f(1)=1$, условия $(2.12),(2.13)$ превращаются в необходимые и достаточные условия монотонности, описанные в теореме 2.4 .

\section{3. Самоподобные функции нулевого спектрального порядка, задаваемые оператором подобия, меняющего ориентацию отрезка}

Как сказано в замечании 2.1, функция, являющаяся неподвижной точкой оператора $G$, не изменится, если операторы $G_{k}$ поменять на операторы $\widetilde{G}_{k}$ при $k \neq \widehat{k}$. А именно, неподвижная функция отображения $G$ на подотрезках $\left[\alpha_{k}, \alpha_{k+1}\right]$ принимает значения, равные $\beta_{k}$ при $k \neq \widehat{k}$, независимо от того, отображения $\widetilde{G}_{k}$ или $G_{k}$ определяют оператор $G$. Поэтому имеет смысл рассматривать отображение $\widetilde{G}_{k}$ только при $k=\widehat{k}$.

3.1. Формулы для приближений самоподобной функции. Воспользовавшись свойством оператора $\widetilde{G}_{\widehat{k}}$ менять ориентацию отрезка $\left[\alpha_{\widehat{k}}, \alpha_{\widehat{k}+1}\right]$, несложно получить формулы для $m$-х приближений самоподобной функции, аналогичные формулам (2.7)-(2.10). Начальная функция также принимается равной тождественно нулю:

$$
\begin{aligned}
& f_{1}(x)=\beta_{k_{1}}, \quad x \in I_{k_{1}}, \quad k_{1}=1,2, \ldots, n, \\
& f_{2}(x)= \begin{cases}\beta_{k_{1}}, & x \in I_{k_{1}}, \quad k_{1} \neq \widehat{k}, \\
d_{\widehat{k}} \beta_{n-k_{2}+1}+\beta_{\widehat{k}}, & x \in I_{\widehat{k}, k_{2}}, \quad k_{2}=1,2, \ldots, n,\end{cases} \\
& f_{3}(x)= \begin{cases}\beta_{k_{1}}, & x \in I_{k_{1}}, \quad k_{1} \neq \widehat{k}, \\
d_{\widehat{k}} \beta_{n-k_{2}+1}+\beta_{\widehat{k}}, & x \in I_{\widehat{k}, k_{2}}, \quad k_{2} \neq \widehat{k}, \\
d_{\widehat{k}}\left(d_{\widehat{k}} \beta_{k_{3}}+\beta_{\widehat{k}}\right)+\beta_{\widehat{k}}, & x \in I_{\widehat{k}, \widehat{k}, k_{3}}, \quad k_{3}=1,2, \ldots, n,\end{cases}
\end{aligned}
$$




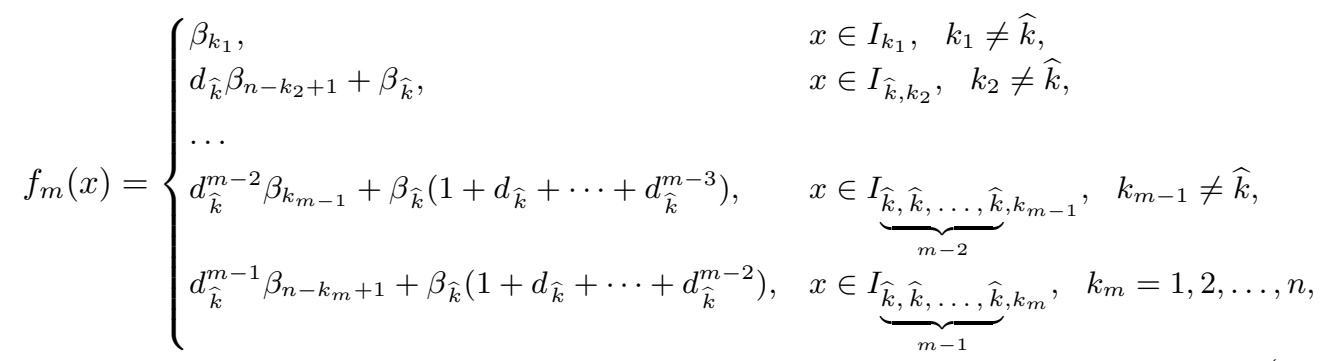

при четном $m$. При нечетном $m$ формула (3.4) принимает вид

$$
f_{m}(x)=\left\{\begin{array}{l}
\beta_{k_{1}}, \\
d_{\widehat{k}} \beta_{n-k_{2}+1}+\beta_{\widehat{k}}, \\
\cdots \\
d_{\widehat{k}}^{m-2} \beta_{n-k_{m-1}+1}+\beta_{\widehat{k}}\left(1+d_{\widehat{k}}+\cdots+d_{\widehat{k}}^{m-3}\right), \\
d_{\widehat{k}}^{m-1} \beta_{k_{m}}+\beta_{\widehat{k}}\left(1+d_{\widehat{k}}+\cdots+d_{\widehat{k}}^{m-2}\right),
\end{array}\right.
$$

$$
\begin{aligned}
& x \in I_{k_{1}}, \quad k_{1} \neq \widehat{k}, \\
& x \in I_{\widehat{k}, k_{2}}, \quad k_{2} \neq \widehat{k}, \\
& x \in \underbrace{I_{\widehat{k}, \widehat{k}, \ldots, \widehat{k}}, k_{m-1}}_{m-2}, \quad k_{m-1} \neq \widehat{k}, \\
& x \in \underbrace{I_{\widehat{k}, \widehat{k}, \ldots, \widehat{k}}, k_{m}}_{m-1}, \quad k_{m}=1,2, \ldots, n .
\end{aligned}
$$

3.2. Координаты особой точки. Рассмотрим, как меняются формулы для определения координат особой точки, если преобразование с номером $\widehat{k}$ меняет ориентацию отрезка $\left[\alpha_{\widehat{k}}, \alpha_{\widehat{k}+1}\right]$. Координаты левого и правого концов отрезка $\underbrace{}_{\frac{\widehat{k}}{\widehat{k}, \widehat{k}}, \ldots, \widehat{k}}$ в этом случае имеют вид

$$
\begin{aligned}
& \widetilde{x}_{l}=\alpha_{\widehat{k}}+a_{\widehat{k}}\left(1-\alpha_{\widehat{k}+1}\right)+a_{\widehat{k}}^{2} \alpha_{\widehat{k}}+a_{\widehat{k}}^{2}\left(1-\alpha_{\widehat{k}+1}\right)+\cdots+a_{\widehat{k}}^{\lceil m / 2\rceil} A, \\
& \widetilde{x}_{r}=1-\left(1-\alpha_{\widehat{k}+1}\right)-a_{\widehat{k}} \alpha_{\widehat{k}}-a_{\widehat{k}}^{2}\left(1-\alpha_{\widehat{k}+1}\right)-a_{\widehat{k}}^{2} \alpha_{\widehat{k}}-\cdots-a_{\widehat{k}}^{\lceil m / 2\rceil} A,
\end{aligned}
$$

где

$$
A= \begin{cases}\alpha_{\widehat{k}} & \text { при } m=2 l-1, \\ 1-\alpha_{\widehat{k}+1} & \text { при } m=2 l,\end{cases}
$$

а $\lceil x\rceil$ - наименьшее целое число, не меньшее $x$ ("потолок"), $l=1,2, \ldots$.

Несложно вычислить, что особая точка $\widehat{x}$ в этом случае имеет координату

$$
\widehat{x}=\frac{\alpha_{\widehat{k}+1}}{1+a_{\widehat{k}}} .
$$

Отметим, что в этом случае, независимо от значения $\widehat{k}$, особая точка $\widehat{x}$ не может совпасть с концами отрезка $[0,1]$.

3.3. Условия неубывания функции в случае $\widetilde{G}_{\widehat{k}}$. Используя формулы $(3.1)$ (3.4), несложно получить аналог теоремы 2.4 .

Теорема 3.1. Самоподобная функиия $f \in D_{1}$, заданная таким оператором подобия, у которого $\widetilde{G}_{\widehat{k}}$ меняет ориентацию отрезка $\left[\alpha_{\widehat{k}}, \alpha_{\widehat{k}+1}\right]$, является неубъвающей тогда и только тогда, когда ее параметры самоподобия удовлетворяют следуюшим условиям: 
1) $d_{\widehat{k}}<0$

2) выполнено

$$
\beta_{1} \leqslant \beta_{2} \leqslant \cdots \leqslant \beta_{\widehat{k}-1} \leqslant d_{\widehat{k}} \beta_{n}+\beta_{\widehat{k}} \leqslant d_{\widehat{k}} \beta_{1}+\beta_{\widehat{k}} \leqslant \beta_{\widehat{k}+1} \leqslant \cdots \leqslant \beta_{n}
$$

npu $1<\widehat{k}<n$.

При $\widehat{k}=n$ условие 2) примет вид

$$
\beta_{1} \leqslant \beta_{2} \leqslant \cdots \leqslant \beta_{n-1} \leqslant \beta_{n}, \quad \beta_{n-1} \leqslant d_{n} \beta_{n}+\beta_{n} .
$$

При $\widehat{k}=1$ условие 2) примет вид

$$
\beta_{1} \leqslant \beta_{2} \leqslant \cdots \leqslant \beta_{n-1} \leqslant \beta_{n}, \quad d_{1} \beta_{1}+\beta_{1} \leqslant \beta_{2} .
$$

ЗАмЕчАниЕ 3.1. Функцию нулевого спектрального порядка, у которой преобразование $\widetilde{G}_{\widehat{k}}$ меняет ориентацию отрезка $\left[\alpha_{\widehat{k}}, \alpha_{\widehat{k}+1}\right]$, можно задать другим набором параметров самоподобия, для которых все преобразования $G_{i}$ сохраняют ориентацию отрезков.

Чтобы найти эти параметры самоподобия, достаточно заметить, что сжимающее отображение $G^{2}$ имеет ту же неподвижную функцию $f$, что и преобразование $G$. Введем преобразование $F:=G^{2}$. Пусть $N, \alpha(F)_{k}, \beta(F)_{k}$ и $d(F)_{k}, k=1,2, \ldots, N,-$ его параметры самоподобия. Через $m$ обозначим тот единственный индекс, для которого $d(F)_{m} \neq 0$. Эти числа можно вычислить через параметры самоподобия исходного преобразования $G$ следующим образом:

$$
\begin{gathered}
N=2 n-1, \quad m=n, \quad d(F)_{m}=d_{\widehat{k}}^{2}, \\
a(F)_{k}=a_{k}, \quad k=1,2, \ldots, \widehat{k}-1, \\
a(F)_{m+k}=a_{\widehat{k}} \cdot a_{n-k}, \quad k=0,1, \ldots, n-1, \\
a(F)_{m+n+k}=a_{\widehat{k}+k+1}, \quad k=0,1, \ldots, n-\widehat{k}, \\
\beta(F)_{k}=\beta_{k}, \quad k=1,2, \ldots, \widehat{k}-1, \\
\beta(F)_{m+k}=d_{\widehat{k}} \cdot \beta_{n-k}, \quad k=0,1, \ldots, n-1, \\
\beta_{m+n+k}=\beta_{\widehat{k}+k+1}, \quad k=0,1, \ldots, n-\widehat{k} .
\end{gathered}
$$

Как и преобразование $G$, преобразование $F$ также можно задать с помощью отображений $F_{k}, k=1,2, \ldots, N$, сопоставляющих функции $f \in L_{p}[0,1]$ функции $F_{k}(f) \in L_{p}\left[\alpha(F)_{k}, \alpha(F)_{k+1}\right]:$

$$
\left(F_{k} f\right)(x)=f(t), \quad x=a(F)_{k} t+\alpha(F)_{k}, \quad x \in\left[\alpha(F)_{k}, \alpha(F)_{k+1}\right], \quad t \in[0,1] .
$$

$\mathrm{У}$ преобразования $F$ все его составляющие преобразования $F_{k}, k=1,2, \ldots, N$, сохраняют ориентацию отрезков.

Автор признателен А. И. Назарову за полезные обсуждения и замечания.

\section{СПИСОК ЦИТИРОВАННОЙ ЛИТЕРАТУРЫ}

[1] И. С. Кац, М. Г. Крейн, "О спектральных функциях струны": Ф. Аткинсон, Дискретные и непрерывные граничные задачи, Мир, М., 1968, 648-733. 
[2] M. Solomyak, E. Verbitsky, "On a spectral problem related to self-similar measures", Bull. London Math. Soc., 27:3 (1995), 242-248.

[3] А. А. Владимиров, И. А. Шейпак, "Самоподобные функции в пространстве $L_{2}[0,1]$ и задача Штурма-Лиувилля с сингулярным индефинитным весом", Матем. сб., 197:11 (2006), 13-30.

[4] А.А. Владимиров, И.А. Шейпак, "Индефинитная задача Штурма-Лиувилля для некоторых классов самоподобных сингулярных весов", Функииональные пространства, теория приближений, нелинейный анализ, Сборник статей, Тр. МИАН, 255, Наука, М., 2006, 88-98.

[5] А.А. Владимиров, И.А. Шейпак, Асимптотика собственных значений задачи Штурма-Лиувилля с дискретным самоподобным весом, arXiv: math.SP/0709.0424.

[6] M. Barnsley, Fractals Everywere, Academic Press, Boston, MA, 1988.

[7] G. de Rham, "Une peu de mathématiques à propos d'une courbe plane", Elem. Math., 2:4-5 (1947), 73-76, 89-97.

[8] G. de Rham, "Sur une courbe plane", J. Math. Pures Appl. (9), 35 (1956), 25-42.

[9] G. de Rham, "Sur les courbes limités de polygones obtenus par trisection", Enseign. Math. (2), 5 (1959), 29-43.

[10] V. Protasov, "Refinement equations with nonnegative coefficients", J. Fourier Anal. Appl., 6:1 (2000), 55-78.

[11] В.Ю. Протасов, “Фрактальные кривые и всплески”, Изв. РАН. Сер. матем., 70:5 (2006), 123-162.

[12] И. А. Шейпак, "О конструкции и некоторых свойствах самоподобных функций в пространствах $L_{p}[0,1]$ ", Матем. заметки, 81:6 (2007), 924-938.

[13] А.И. Назаров, “Логарифмическая асимптотика малых уклонений для некоторых гауссовских случайных процессов в $L_{2}$-норме относительно самоподобной меры”, $B e$ роятность и статистика. 7, Зап. научн. сем. ПОМИ, 311, ПОМИ, СПб., 2004, $190-213$.

И. А. Шейпак

Московский государственный университет

им. М. В. Ломоносова

E-mail: iasheip@mech.math.msu.su
Поступило

16.06.2008 